\title{
A Comparison of Parallel Profiling Tools for Programs utilizing the FFT
}

\author{
Brian Leu \\ brian.d.leu@gmail.com \\ Applied Dynamics International \\ Ann Arbor, Michigan, USA
}

\author{
Samar Aseeri \\ King Abdullah University of Science \\ and Technology \\ Thuwal, Saudi Arabia \\ samar.aseeri@kaust.edu.sa
}

\author{
Benson K. Muite \\ Kichakato Kizito \\ Nairobi, Kenya \\ benson_muite@emailplus.org
}

\begin{abstract}
Performance monitoring is an important component of code optimization. Performance monitoring is also important for the beginning user, but can be difficult to configure appropriately. The overhead of the performance monitoring tools Craypat, FPMP, mpiP, Scalasca and TAU, are measured using default configurations likely to be choosen by a novice user and shown to be small when profiling Fast Fourier Transform based solvers for the Klein Gordon equation based on 2decomp\&FFT and on FFTE. Performance measurements help explain that despite FFTE having a more efficient parallel algorithm, it is not always faster than 2decom\&FFT because the complied single core FFT is not as fast as that in FFTW which is used in 2 decomp\&FFT.
\end{abstract}

\section{CCS CONCEPTS}

- Computing methodologies $\rightarrow$ Massively parallel algorithms; - Networks $\rightarrow$ Network measurement.

\section{KEYWORDS}

Performance, Profiling Tools, Fast Fourier Transform

\section{ACM Reference Format:}

Brian Leu, Samar Aseeri, and Benson K. Muite. 2021. A Comparison of Parallel Profiling Tools for Programs utilizing the FFT. In IXPUG Workshop HPC Asia '21: International Conference on High Performance Computing in Asia-Pacific Region, January 20-22, 2021, feju, South Korea. ACM, New York, NY, USA, 10 pages. https://doi.org/10.1145/1122445.1122456

\section{INTRODUCTION}

Performance analysis of parallel programs can be greatly aided by using profiling tools. It can take time to learn how to use all available profiling tools, thus it is helpful to know what the different profiling tools can do. When first learning how to use distributed memory parallel computing systems, profiling tools can give the programmer insight into how well their program works, and the bottlenecks preventing good performance. The main motivation for the study is that IPM is a lightweight, portable and easy to use profiling tool that can be introduced at the early stages of teaching

Permission to make digital or hard copies of all or part of this work for personal or classroom use is granted without fee provided that copies are not made or distributed for profit or commercial advantage and that copies bear this notice and the full citation on the first page. Copyrights for components of this work owned by others than ACM must be honored. Abstracting with credit is permitted. To copy otherwise, or republish, to post on servers or to redistribute to lists, requires prior specific permission and/or a fee. Request permissions from permissions@acm.org.

IXPUG Workshop HPC Asia '21, fanuary 20-22, 2021, Jeju, South Korea

(c) 2021 Association for Computing Machinery.

ACM ISBN 978-1-4503-XXXX-X/18/06 .. \$15.00

https://doi.org/10.1145/1122445.1122456 distributed memory parallel programming. It produces a nice text summary, with an optional graphical webpage. It can also be run in production settings, for example to supplement supercomputer center job profiling information[35]. However, it primarily profiles point to point communication and does not profile MPI_ALLTOALL which is the dominant communication call in many Fast Fourier Transform programs. The aim is therefore to find a suitable easy to use portable replacement for IPM that captures information about MPI_ALLTOALL. It is also of interest to compare portable open software to vendor provided tools. On systems with Dragonfly networks that have adaptive routing and opportunistic job placement, job interference can result in dramatic changes to program execution time - it is unclear what the signature of such interference will be when profiling with the different tools. A final aim is to see if profiling can help provide an explanation for the differences in scaling behavior of FFTE as compared to 2decomp\&FFT.

The tools, IPM, Scalasca, CrayPat, mpiP, FPMPI and TAU are compared when profiling a Fast Fourier Transform solvers for the nonlinear Klein Gordon equation. The main findings are:

- Some of these tools can be challenging to setup if not already setup for the user

- For the number of cores considered here, these tools can be used in a production setting for low overhead profiling

- Vendor provided tools can allow for well integrated hardware performance monitoring that may not be otherwise easily exposed or integrated in the portable open source tools

\section{TEST PROGRAM}

Previous work has compared the performance of a Fast Fourier Transform (FFT) based solver for the three dimensional KleinGordon equation[3] using 2decomp\&FFT. That study primarily focused on measuring time to solution and strong scaling results. The study also produced a simple performance model. More detailed performance models can be validated using performance measurements. In this study the numerical solution of the Klein-Gordon equation is again used as an example mini-application for which performance profiling tools can also be compared. In addition, a solver using the FFT library FFTE is included in the comparison. Earlier measurements shown in Fig. 1 showed that TAU[7, 30, 31, 42], a performance profiling tool can be configured to have low overhead even when profiling at large core counts. One of the aims of this work is to quantify the level of overhead and compare TAU to other profiling tools. 


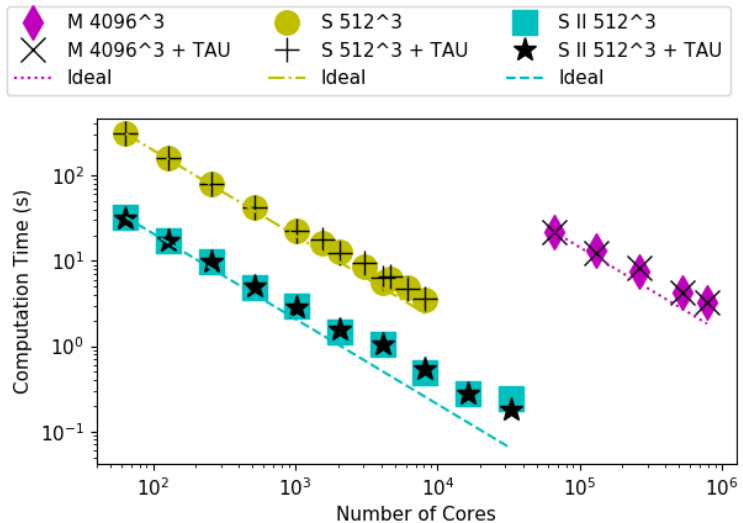

Figure 1: Strong scaling and overhead of profiling with TAU for 30 timesteps of the Klein-Gordon equation at spatial resolutions of $512^{3}$ and $4096^{3}$ grid points on Mira (M), Shaheen (S), and Shaheen II (S II).

\section{PROFILING TOOLS, FFT LIBRARY AND TEST PLATFORMS}

\subsection{The Profiling Tools}

A summary of parallel program profiling tools is given in Table 1 . Here, a general introduction to profiling tools is given, and then more detailed information on the profiling tools used in this study follows.

A number of the profiling tools used are open source (Scalsaca, TAU, IPM, mpiP and FPMPI) and if not installed on a machine, require the user to install them. Some of these tools share components (in particular Scalasca and TAU). Many of the open source tools (such as Scalasca, and TAU) are also performance monitoring research testbeds, thus they develop quite rapidly. The open source tools tend to rely on very similar components, in particular, binutils, dyninst, libunwind and Papi.

Craypat is a vendor produced profiling tool. The developers can therefore make a tradeoff on configurability by the user, for ease of use and can take advantage of special hardware features. As an example, at present, there is no standard interface to measure energy use or input and output utilization (although there are efforts towards this such as Power API[20], the Global Extensible Open Power Manager[14], Energy Aware Runtime systems[11] and PowerAPI[10]) but Craypat can provide this information to the user while the open source tools that can do this, TAU and Extrae, need to be configured to do so.

3.1.1 IPM. [16, 48] is a lightweight profiling tool meant to be easy to use and display performance information that can also be collected at runtime. It primarily obtains performance information about MPI calls (primarily point to point communication), and can also obtain information about floating point operations if configured to obtain information using Papi[45].

3.1.2 Craypat. is part of the Cray parallel programming productivity suite. It comes installed on Cray computers and is directly integrated on each computer to enable ease of use and to take advantage of hardware features available on the computer. Craypat has a light profiling mode that allows the user to profile code without doing manual instrumentation and to enable for performance improvement by profiling program execution and then using the profiling information to improve subsequent program executions, for example by rank re-ordering. Craypat can instrument executables produced by GNU, Cray, PGI and Intel compilers. A previous study of Craypat is in [41].

Associated with Craypat is a desktop/laptop visualization package, Reveal.

3.1.3 FPMPI. [21] is an open source lightweight profiling tool that primarily targets MPI functions. It is no longer under active development, but is lightweight and still builds on modern CPU only architectures.

3.1.4 mpiP. [47] is an open source lightweight MPI call profiling library. It profiles most of the MPI 2 and MPI 3 calls, not just point to point communications as is done by IPM. Many of its developers are currently developing Openspeedshop.

3.1.5 Scalasca. [17] is a parallel profiling and tracing toolkit developed primarily by FZ Jülich. It offers an incremental performance analysis procedure that integrates runtime summaries with in-depth studies of concurrent behavior. It primarily traces and profiles $\mathrm{C}, \mathrm{C}++$ and Fortran programs. In the experiments reported here Score- $\mathrm{P}[26]$ was used for the instrumentation. It also has an associated performance visualization tool, Cube.

3.1.6 TAU. [7, 30, 31, 42] is an open source tracing and profiling tool that is developed and maintained by the University of Oregon and ParaTools, Inc. It has the capability to instrument Fortran, $\mathrm{C}$ and $\mathrm{C}++$ programs using OpenMP, MPI, Cuda and OpenCL. It has also had the capability of instrumenting and profiling Java and Python programs. While TAU can do binary instrumentation, in typical use, TAU will be setup to perform specific profiling or tracing by instrumenting and then compiling the instrumented source code. This minimizes extra overhead and allows a mapping of the function calls in the output. Good use of TAU requires that the tool user have a good understanding of both the code being studied as well as of TAU. TAU also has typical setup options for tracing and profiling parallel programs. These can be setup and installed for general use. In order to instrument a program using TAU one has to first compile it for each compiler separately so that the produced executable together with the specified parameter options can be used for the profiling instrumentation. It also has an associated visualization tool Paraprof.

A default profiling and tracing experiment will generate a file per process. For a large number of nodes, this can generate a significant amount of data.

\subsection{The Parallel FFT Libraries}

3.2.1 2decomp\&FFT. [29] is a parallel three dimensional Fortran FFT library. It uses external one dimensional FFT libraries and focuses on providing transpose routines. In this study FFTW[15] was used for the one dimensional FFT. The present study used version 1.5 of 2 decomp\&FFT. 
Table 1: An overview of parallel performance tools.

\begin{tabular}{|c|c|c|c|c|c|c|}
\hline Tool & Profiling & Tracing & Open Source & Development & Support & Forum \\
\hline CrayPat[12] & Yes & Yes & No & Yes & Paid & No \\
\hline Extrae[6] & Yes & Yes & Yes & Active & Yes & No \\
\hline FPMPI[21] & Yes & Yes & Yes & No & No & No \\
\hline Hpctoolkit[1] & Yes & Yes & Yes & Active & Paid & Open \\
\hline IPM[16, 48] & Yes & No & Yes & Maintain & No & No \\
\hline mpiP[47] & Yes & No & Yes & Very little & No & Open \\
\hline Openspeedshop[27] & Yes & Yes & Yes & Yes & Paid & Closed \\
\hline PAPIex[33] & Yes & No & Yes & Yes & No & No \\
\hline Scalasca[17] & Yes & Yes & Yes & Active & Free best effort & No \\
\hline TAU[7, 30,31,42] & Yes & Yes & Yes & Active & Paid & Open \\
\hline Vtune[23] & Yes & Yes & No & Yes & Paid & Open \\
\hline
\end{tabular}

3.2.2 FFTE. $[43,44]$ is a Fortran library and for parallel one, two and three dimensional FFTs. The MPI-parallel version only works correctly when the number of MPI processes divides the grid dimensions. The present study used version 7.0 of FFTE.

\subsection{Test Platforms}

3.3.1 Shaheen. [46] was an IBM Blue Gene P supercomputer at the King Abdullah University of Science and Technology. It had IBM PowerPC 4504 core $0.85 \mathrm{GHz}$ processors, a 3D torus network and 65,536 compute cores. This machine was used for the initial studies.

3.3.2 Mira. [2] was an IBM Blue Gene Q supercomputer at the Argonne National Laboratory. It had IBM PowerPC A2 16 core 1.6GHz processors, a 5D torus network and 786,432 compute cores. This machine was used for the initial studies.

3.3.3 Shaheen II. [25] is a Cray XC-40 supercomputer at the King Abdullah University of Science and Technology. It has Intel Xeon E52698v3 16C 2.3GHz processors, a Dragonfly network interconnect and 197,568 compute cores.

\section{PROFILING OVERHEAD MEASUREMENTS}

The supplementary materials[28] includes procedures for installing IPM, TAU, mpiP, FPMPI and Scalasca on Shaheen II, some profiler outputs and code for reproducing the results. In the appendix, there are typical profiler outputs from TAU, Scalasca, Craypat, mpiP and FPMPI for both FFTE and 2decomp\&FFT. When profiilng the Klein Gordon code using 2decomp\&FFT, 2decomp\&FFT was first compiled on its own with instrumentation, the Klein Gordon code using 2decomp\&FFT was then compiled with instrumentation and using the corresponding instrumented 2decom\&FFT library. The Klein Gordon code using FFTE integrated FFTE directly, and so both FFTE and the Klein Gordon code were compiled with instrumentation at the same time. In all cases GCC compilers were used, Cray and Intel compilers do show some performance improvements of upto $4 \%$ in time to solution for the uninstrumented programs on a single node, but as configuring each profiling tool with a range of compilers is time consuming, and GCC is highly portable, GCC was choosen as the compiler to do the measurements on. Profiling with TAU was done using tau f90.sh with optimization -O3 to compile and then running the code normally (for example for 64 cores using srun - $n$ 64.(executable), since TAU by default does lightweight profiling. Profiling with Scalasca was done using scorep $f t n$ to compile and then running the code, for example for 64 cores with scalasca -analyze srun - $n$ 64. .executable or scan srun - $n$ 64. .executable. Profiling with Craypat-lite was done using $f t n$ with optimization -O3 to compile and then running the code normally (for example for 64 cores using srun $-n$ 64. (executable). Profiling with mpiP was done using $f t n$ with optimization -O3 to compile and link to the installed mpiP library, then running the code, for example for 64 cores using srun $-n$ 64. /executable. Profiling with FPMPI was done using $f t n$ with optimization -O3 to compile and link to the FPMPI library, and then running the code, for example for 64 cores using srun - $n 64$ .executable. Only compiler flags were used to optimize the code.

Performance profiling measurements are presented in Table 2 for 30 timesteps of the numerical solution of the Klein-Gordon equation as done in [3]. Each measurement is repeated three times to give confidence in reproducibility, as suggested for example in [4]. On large core counts, this can result in wall clock times significantly less than 10 seconds. A wall clock time of 10 seconds is a good compromise between confidence that the supercomputer results are representative of typical cases and not using limited core hours only for performance measurement. In this study, the allocated available core hours were spent on setting up the tests and ensuring their correctness, so it was not possible to use wall clock times of at least 10 seconds on larger core counts. Table 2 compares Craypat-lite, Scalasca, TAU, mpiP and FPMPI on Shaheen II for a $512^{3}$ discretization with 2decomp\&FFT.

Table 2 shows that the overhead of profiling is within the noise obtained when reproducing the experiments. It also shows that FFTE and 2decomp\&FFT have comparable levels of performance for an FFT of size $512^{3}$ but with increasing core counts FFTE is typically faster than 2decomp\&FFT because it uses fewer MPI_ALLTOALL calls than 2decomp\&FFT. At lower core counts, transpositions are less costly and data alignment that allows the use of optimized instructions available in FFTW make the performance of 2decomp\&FFT similar to that in FFTE. The reason FFTE uses fewer MPI_ALLTOALL calls is because in the fastest MPI parallel implementation of FFTE, the Fourier modes are not stored in a sorted order, requiring some programmer effort to make use of the library. 
Table 2: Table comparing execution times for 30 time steps of FFT based Klein Gordon equation solvers for a $512^{3}$ problem size using 2decomp\&FFT with FFTW and using FFTE libraries. Instrumentation has been done with the Craypat-lite, Scalasca, TAU, mpiP and FPMPI tools on Shaheen II. The mean and standard deviation from 3 runs are shown.

\begin{tabular}{|c|c|c|c|c|c|c|c|}
\hline Cores & FFT & No profiling & Craypat-lite & Scalasca & TAU & mpiP & FPMPI \\
\hline 64 & 2decomp\&FFT & $32.54 \pm 0.10 \mathrm{~s}$ & $31.92 \pm 0.38 \mathrm{~s}$ & $25.35 \pm 0.02 \mathrm{~s}$ & $31.72 \pm 0.50 \mathrm{~s}$ & $31.67 \pm 0.10 \mathrm{~s}$ & $31.97 \pm 0.41 \mathrm{~s}$ \\
\hline 64 & FFTE & $30.68 \pm 0.05 \mathrm{~s}$ & $30.69 \pm 0.03 \mathrm{~s}$ & $30.67 \pm 0.01 \mathrm{~s}$ & $30.45 \pm 0.36 \mathrm{~s}$ & $29.90 \pm 0.33 \mathrm{~s}$ & $31.11 \pm 0.05 \mathrm{~s}$ \\
\hline 128 & 2decomp\&FFT & $17.43 \pm 0.17 \mathrm{~s}$ & $17.57 \pm 0.24 \mathrm{~s}$ & $17.72 \pm 0.94 \mathrm{~s}$ & $17.45 \pm 0.17 \mathrm{~s}$ & $17.64 \pm 0.03 \mathrm{~s}$ & $17.33 \pm 0.07 \mathrm{~s}$ \\
\hline 128 & FFTE & $17.76 \pm 0.03 \mathrm{~s}$ & $18.26 \pm 0.08 \mathrm{~s}$ & $18.89 \pm 0.001 \mathrm{~s}$ & $18.06 \pm 0.16 \mathrm{~s}$ & $18.62 \pm 0.02 \mathrm{~s}$ & $18.17 \pm 0.09 \mathrm{~s}$ \\
\hline 256 & 2decomp\&FFT & $9.72 \pm 0.26 \mathrm{~s}$ & $9.71 \pm 0.03 \mathrm{~s}$ & $10.20 \pm 0.07 \mathrm{~s}$ & $9.67 \pm 0.01 \mathrm{~s}$ & $9.88 \pm 0.23 \mathrm{~s}$ & $9.75 \pm 0.06 \mathrm{~s}$ \\
\hline 256 & FFTE & $9.73 \pm 0.02 s$ & $9.73 \pm 0.01 \mathrm{~s}$ & $9.81 \pm 0.01 \mathrm{~s}$ & $9.78 \pm 0.06 \mathrm{~s}$ & $9.70 \pm 0.01 \mathrm{~s}$ & $9.93 \pm 0.22 \mathrm{~s}$ \\
\hline 512 & 2decomp\&FFT & $4.93 \pm 0.07 s$ & $5.17 \pm 0.26 \mathrm{~s}$ & $5.12 \pm 0.04 \mathrm{~s}$ & $5.04 \pm 0.13 \mathrm{~s}$ & $5.15 \pm 0.17 \mathrm{~s}$ & $5.01 \pm 0.03 \mathrm{~s}$ \\
\hline 512 & FFTE & $5.09 \pm 0.03 \mathrm{~s}$ & $4.99 \pm 0.21 \mathrm{~s}$ & $4.97 \pm 0.03 \mathrm{~s}$ & $4.91 \pm 0.04 \mathrm{~s}$ & $4.86 \pm 0.13 \mathrm{~s}$ & $4.76 \pm 0.01 \mathrm{~s}$ \\
\hline 1024 & 2decomp\&FFT & $2.93 \pm 0.02 \mathrm{~s}$ & $3.15 \pm 0.29 \mathrm{~s}$ & $2.87 \pm 0.06 \mathrm{~s}$ & $2.89 \pm 0.03 \mathrm{~s}$ & $3.10 \pm 0.10 \mathrm{~s}$ & $2.92 \pm 0.03 \mathrm{~s}$ \\
\hline 1024 & FFTE & $2.48 \pm 0.03 \mathrm{~s}$ & $2.24 \pm 0.02 \mathrm{~s}$ & $2.59 \pm 0.29 \mathrm{~s}$ & $2.24 \pm 0.01 \mathrm{~s}$ & $2.21 \pm 0.05 \mathrm{~s}$ & $2.30 \pm 0.08 \mathrm{~s}$ \\
\hline 2048 & 2decomp\&FFT & $1.45 \pm 0.017 \mathrm{~s}$ & $1.67 \pm 0.05 \mathrm{~s}$ & $1.64 \pm 0.02 \mathrm{~s}$ & $1.57 \pm 0.03 \mathrm{~s}$ & $1.51 \pm 0.13 \mathrm{~s}$ & $1.86 \pm 0.20 \mathrm{~s}$ \\
\hline 2048 & FFTE & $1.30 \pm 0.10 \mathrm{~s}$ & $1.62 \pm 0.45 \mathrm{~s}$ & $1.29 \pm 0.07 \mathrm{~s}$ & $1.26 \pm 0.02 \mathrm{~s}$ & $1.19 \pm 0.03 \mathrm{~s}$ & $1.67 \pm 0.51 \mathrm{~s}$ \\
\hline 4096 & 2decomp\&FFT & $1.04 \pm 0.03 \mathrm{~s}$ & $1.01 \pm 0.04 \mathrm{~s}$ & $0.91 \pm 0.03 \mathrm{~s}$ & $1.05 \pm 0.08 \mathrm{~s}$ & $0.93 \pm 0.06 \mathrm{~s}$ & $0.91 \pm 0.04 \mathrm{~s}$ \\
\hline 4096 & FFTE & $0.66 \pm 0.03 \mathrm{~s}$ & $0.659 \pm 0.002 \mathrm{~s}$ & $0.64 \pm 0.02 \mathrm{~s}$ & $0.63 \pm 0.01 \mathrm{~s}$ & $0.67 \pm 0.03 \mathrm{~s}$ & $0.70 \pm 0.01 \mathrm{~s}$ \\
\hline 8192 & 2decomp\&FFT & $0.487 \pm 0.002 \mathrm{~s}$ & $0.50 \pm 0.02 \mathrm{~s}$ & $0.54 \pm 0.02 \mathrm{~s}$ & $0.54 \pm 0.02 \mathrm{~s}$ & $0.56 \pm 0.11 \mathrm{~s}$ & $0.49 \pm 0.02 \mathrm{~s}$ \\
\hline 8192 & FFTE & $0.50 \pm 0.01 \mathrm{~s}$ & $0.53 \pm 0.05 \mathrm{~s}$ & $0.53 \pm 0.10 \mathrm{~s}$ & $0.47 \pm 0.02 \mathrm{~s}$ & $0.48 \pm 0.03 \mathrm{~s}$ & $0.51 \pm 0.01 \mathrm{~s}$ \\
\hline 16384 & 2decomp\&FFT & $0.28 \pm 0.01 \mathrm{~s}$ & $0.27 \pm 0.01 \mathrm{~s}$ & $0.35 \pm 0.08 \mathrm{~s}$ & $0.28 \pm 0.01 \mathrm{~s}$ & $0.28 \pm 0.01 \mathrm{~s}$ & $0.28 \pm 0.02 \mathrm{~s}$ \\
\hline 16384 & FFTE & $0.255 \pm 0.002 \mathrm{~s}$ & $0.26 \pm 0.02$ & $0.33 \pm 0.06 \mathrm{~s}$ & $0.28 \pm 0.03 \mathrm{~s}$ & $0.30 \pm 0.10 \mathrm{~s}$ & $0.30 \pm 0.04 \mathrm{~s}$ \\
\hline 32768 & 2decomp\&FFT & $0.24 \pm 0.04 \mathrm{~s}$ & $0.22 \pm 0.04 \mathrm{~s}$ & $0.25 \pm 0.06 \mathrm{~s}$ & $0.18 \pm 0.01 \mathrm{~s}$ & $0.21 \pm 0.03 \mathrm{~s}$ & $0.25 \pm 0.06 \mathrm{~s}$ \\
\hline 32768 & FFTE & $0.22 \pm 0.04 \mathrm{~s}$ & $0.18 \pm 0.02$ & $0.19 \pm 0.01 \mathrm{~s}$ & $0.16 \pm 0.01 \mathrm{~s}$ & $0.31 \pm 0.21 \mathrm{~s}$ & $0.18 \pm 0.02 \mathrm{~s}$ \\
\hline
\end{tabular}

\section{DISCUSSION}

This study has primarily focused on measuring the ease of use and overhead of different performance profiling tools. The algorithms used in the tools and their implementation determine the amount of overhead, but the typical user of the tools is primarily interested in the measurement and its associated cost, rather than an in depth performance tool developers knowledge of the tools.

One can consider a derived overhead metric of a tool as the ratio of the computation time without profiling to that with profiling. Overheads are presented in Figures 2a and 2b for Mira, Shaheen and Shaheen II respectively. Measurements on Mira and Shaheen were not repeated multiple times, and since these are no longer operational, it is not possible to repeat the measurements. The torus networks on Mira and Shaheen isolate communication interference between running programs, but this does not occur on Shaheen II, which has more variability for time to solution of communication intensive programs. The figures show that default configurations of the used profiling tools have low overhead for the measured core counts for an application which primarily has collective communication calls. While the profiling tools can give information about job placement, in dragonfly networks, they do not give information about communication interference due to other jobs, such information may be helpful in job placement, job scheduling and in modifying routing communication algorithms. It may also be of help in evaluating network designs such as the Slim Fly topology[8] for the effects of job interference on variability in time to completion of communication intensive programs running on a portion of a supercomputer.

Mira and Shaheen have different types of torus networks, with Shaheen having processors about half as fast as Mira. Figures 2a and 1 show that when one considers grid points per core, scaling on Shaheen is better than Mira because the processor speed, memory bandwidth and network bandwidth on Shaheen are in better balance than on Mira. Similarly, Figure 1 shows that the parallel efficiency at high core counts on Shaheen II is not as good as Shaheen, nevertheless, the time to solution on Shaheen II is less than on Shaheen.

Craypat-lite and FPMPI automatically provide short summary text files with aggregate information at the end of each profiling experiment. mpiP also provides a summary text file, however this contains node level information, which while useful for understanding performance imbalance, can become quite lengthy when using thousands of cores. Scalasca, TAU and Craypat-lite also produce comprehensive measurement files that can be postprocessed to obtain further information. For Scalasca and TAU, a postprocessing step is needed after job completion to obtain a summary file with aggregate job information. In a production setting, it may be helpful to do this automatically. The resulting profiling information can be used to suggest code improvements as well as monitor the health of the parallel computer. For the current experiments, the summary file provided by FPMPI was the simplest to use as it gave the number of MPI_ALLTOALL calls which are most useful when understanding the performance of communication in the parallel FFT, which dominates the wall clock time. TAU, Scalasca and Craypat-lite also provide useful information on MPI_ALLTOALL to allow one to determine the reasons for the performance differences between the solver using FFTE and the solver using 2decomp\&FFT. The summary information from mpiP is less useful for this as it is aggregated by MPI rank, rather than by MPI call. 


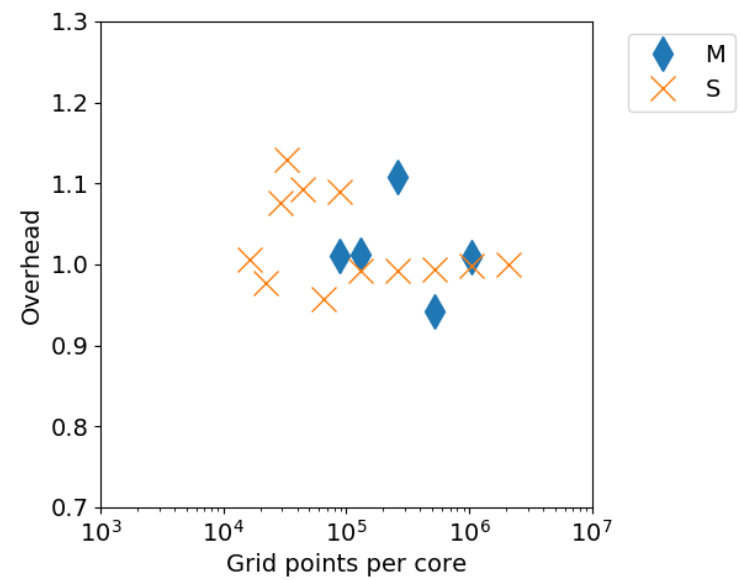

(a) Calculated overhead of profiling with TAU for 30 timesteps of the Klein-Gordon equation on Mira (M) and Shaheen (S) for $4096^{3}$ and $512^{3}$ grids respectively.

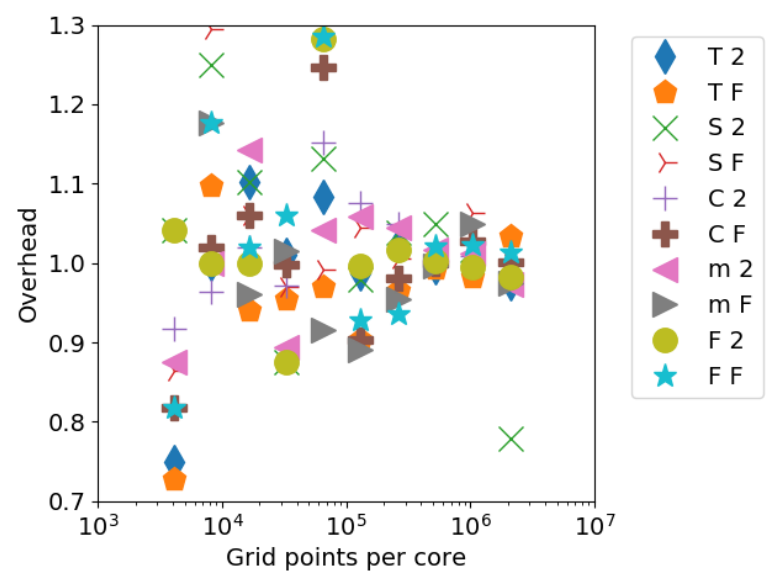

(b) Calculated overhead of profiling with Klein Gordon solver for 30 timesteps on Shaheen II. The first character in the legend indicates the profiling tool, C: Craypat-lite, F: FPMPI, m: mpiP and T: TAU. The second character in the legend indicates the FFT library, F: FFTE and 2: 2decomp\&FFT

Figure 2: Overhead and differences in repeatability on torus (Mira and Shaheen) and dragonfly (Shaheen II) networks

\section{SUMMARY}

Craypat-lite, Scalasca, mpiP, FPMPI and TAU offer reasonable summarized default lightweight profiling options for parallel programs which can be obtained by compiling the programs with appropriate wrappers. Reports produced using Craypat-lite have the most comprehensive information, though it is also possible to obtain more comprehensive information from TAU and Scalasca by changing the runtime configuration. On the core counts used here, all these programs have low enough overhead to be used in a production setting, allowing for monitoring of program performance. In parallel programs, a call graph of function information or timing of MPI routines is provided by most of the tools. Learning to use these is a transferable skill. The vendor provided performance tools may be more comprehensive and are automatically configured for the user, but since users typically use more than one kind of computer, the open source tools are preferable for initial training. For the beginning user, it is helpful to pre-install a lightweight low overhead configuration of a productivity tool for production setting use.

\section{FURTHER WORK}

Further work involves profiling other parallel FFT libraries, such as [5], [9], [13], [18], [19], [24], [32], [36], [37], [38], [39] and [40]. In addition profiling and tracing tools such as Extrae, PapiEx, Intel Vtune and OpenSpeedshop will be included in a more detailed comparison. The effects of job placement and communication interference on result reproducibility will also be studied, an area for which there are still challenges[22]. Finally, there are other numerical methods that can be used to solve the Klein Gordon equation[34], it would be interesting to use these other methods to aid in performance prediction and optimal matching of algorithm to hardware architecture.

\section{ACKNOWLEDGMENTS}

We thank José Gracia, Chirstoph Niethammer, Sameer Shende, Daisuke Takahashi and Brian Wylie for helpful discussions. B.L.'s work was primarily done while affiliated with the University of Michigan. B.K.M. was partially supported by HPC Europa 3 (INFRAIA2016-1-730897) and the Estonian Center for Excellence in IT (TK148), and his work was mostly done while affiliated with the Institute of Computer Science at the University of Tartu. The computational resources used to build and test the programs were:

- Shaheen and Shaheen II operated by the KAUST Supercomputing Laboratory.

- Hazelhen at HLRS.

- K computer that was operated by RIKEN.

- Rocket and Vedur operated by the University of Tartu HPC center.

- Mira that was operated by the Argonne Leadership Computing Facility, which is a DOE Office of Science User Facility supported under Contract DE-AC02-06CH11357.

\section{REFERENCES}

[1] L. Adhianto, S. Banerjee, M. Fagan, M. Krentel, G. Marin, J. Mellor-Crummey, and N. R. Tallent. 2010. HPCTOOLKIT: Tools for performance analysis of optimized parallel programs. Concurrency and Computation: Practice and Experience 22, 6 (2010), 685-701. https://doi.org/10.1002/cpe.1553

[2] Argonne National Laboratory. 2020. Mira. https://www.alcf.anl.gov/alcfresources/mira

[3] S. Aseeri, O. Batrasev, M. Icardi, B. Leu, A. Liu, N. Li, B. K. Muite, E. Müller, B. Palen, M. Quell, H. Servat, P. Sheth, R. Speck, M. Van Moer, and J. Vienne. 2015. Solving the Klein-Gordon Equation Using Fourier Spectral Methods: A Benchmark Test for Computer Performance. In Proceedings of the Symposium on High Performance Computing (Alexandria, Virginia) (HPC '15). Society for Computer Simulation International, San Diego, CA, USA, 182-191. http://dl. acm.org/citation.cfm?id=2872599.2872622

[4] Samar Aseeri, Benson K. Muite, and Daisuke Takahashi. 2019. Reproducibility in Benchmarking Parallel Fast Fourier Transform Based Applications. In Companion of the 2019 ACM/SPEC International Conference on Performance Engineering (Mumbai, India) (ICPE '19). Association for Computing Machinery, New York, NY, USA, 5-8. https://doi.org/10.1145/3302541.3313105

[5] Alan Ayala, Stanimire Tomov, Azzam Haidar, and Jack Dongarra. 2020. heFFTe: Highly Efficient FFT for Exascale. Springer Berlin Heidelberg, Berlin, Heidelberg. https://doi.org/10.1007/978-3-030-50371-0_19 
[6] Barcelona Supercomputing Center. 2019. Extrae instrumentation package. https: //tools.bsc.es/extrae

[7] David E. Bernholdt, Benjamin A. Allan, Robert Armstrong, Felipe Bertrand, Kenneth Chiu, Tamara L. Dahlgren, Kostadin Damevski, Wael R. Elwasif, Thomas G. W. Epperly, Madhusudhan Govindaraju, Daniel S. Katz, James A. Kohl, Manoj Krishnan, Gary Kumfert, J. Walter Larson, Sophia Lefantzi, Michael J. Lewis, Allen D. Malony, Lois C. Mclnnes, Jarek Nieplocha, Boyana Norris, Steven G. Parker, Jaideep Ray, Sameer Shende, Theresa L. Windus, and Shujia Zhou. 2006. A Component Architecture for High-Performance Scientific Computing. The International fournal of High Performance Computing Applications 20, 2 (2006), 163-202. https://doi.org/10.1177/1094342006064488

[8] M. Besta and T. Hoefler. 2014. Slim Fly: A Cost Effective Low-Diameter Network Topology. In SC '14: Proceedings of the International Conference for High Performance Computing, Networking, Storage and Analysis. 348-359. https: //doi.org/10.1109/SC.2014.34

[9] Anando G. Chatterjee, Mahendra K. Verma, Abhishek Kumar, Ravi Samtaney, Bilel Hadri, and Rooh Khurram. 2018. Scaling of a Fast Fourier Transform and a pseudo-spectral fluid solver up to 196608 cores. F. Parallel and Distrib. Comput. 113 (2018), 77 - 91. https://doi.org/10.1016/j.jpdc.2017.10.014

[10] Maxime Colmant, Romain Rouvoy, Mascha Kurpicz, Anita Sobe, Pascal Felber, and Lionel Seinturier. 2018. The Next 700 CPU Power Models. Journal of Systems and Software 144 (July 2018), 382-396. https://doi.org/10.1016/j.jss.2018.07.001

[11] Julita Corbalan and Luigi Brochard. 2019. EAR: Energy management framework for supercomputers. https://www.bsc.es/sites/default/files/public/bscw2/ content/software-app/technical-documentation/ear.pdf

[12] Cray. 2020. Cray Performance Measurement and Analysis Tools User Guide (7.0.0) S-2376. https://pubs.cray.com/content/S-2376/7.0.0/cray-performancemeasurement-and-analysis-tools-user-guide/craypat

[13] Lisandro Dalcin, Mikael Mortensen, and David E. Keyes. 2019. Fast parallel multidimensional FFT using advanced MPI. f. Parallel and Distrib. Comput. 128 (2019), 137 - 150. https://doi.org/10.1016/j.jpdc.2019.02.006

[14] Jonathan Eastep, Steve Sylvester, Christopher Cantalupo, Brad Geltz, Federico Ardanaz, Asma Al-Rawi, Kelly Livingston, Fuat Keceli, Matthias Maiterth, and Siddhartha Jana. 2017. Global Extensible Open Power Manager: A Vehicle for HPC Community Collaboration on Co-Designed Energy Management Solutions. In High Performance Computing, Julian M. Kunkel, Rio Yokota, Pavan Balaji, and David Keyes (Eds.). Springer International Publishing, Cham, 394-412.

[15] M. Frigo and S. G. Johnson. 2005. The Design and Implementation of FFTW3. Proc IEEE 93, 2 (2005), 216-231. Special issue on "Program Generation, Optimization, and Platform Adaptation".

[16] Karl Fürlinger, Nicholas J. Wright, and David Skinner. 2010. Performance Analysis and Workload Characterization with IPM. In Tools for High Performance Computing 2009, Matthias S. Müller, Michael M. Resch, Alexander Schulz, and Wolfgang E. Nagel (Eds.). Springer Berlin Heidelberg, Berlin, Heidelberg, 31-38.

[17] Markus Geimer, Felix Wolf, Brian J. N. Wylie, Erika Âbrahám, Daniel Becker, and Bernd Mohr. 2010. The Scalasca performance toolset architecture. Concurrency and Computation: Practice and Experience 22, 6 (2010), 702-719. https://doi.org/ 10.1002/cpe.1556

[18] A. Gholami, J. Hill, D. Malhotra, and G. Biros. 2016. AccFFT: A library for distributed-memory FFT on CPU and GPU architectures,. http://arxiv.org/abs/ 1506.07933

[19] J.H. Göbbert. 2017. nb3dfft. https://gitlab.version.fz-juelich.de/goebbert/nb3dfft

[20] R. E. Grant, M. Levenhagen, S. L. Olivier, D. DeBonis, K. T. Pedretti, and J. H. Laros III. 2016. Standardizing Power Monitoring and Control at Exascale. Computer 49, 10 (Oct 2016), 38-46. https://doi.org/10.1109/MC.2016.308

[21] William Gropp and Kristopher Buschelman. 2020. FPMPI. https://www.mcs.anl. gov/research/projects/fpmpi/WWW/index.html

[22] Sascha Hunold, Alexandra Carpen-Amarie, and Jesper Larsson Träff. 2014 Reproducible MPI Micro-Benchmarking Isn't As Easy As You Think. In Proceedings of the 21st European MPI Users' Group Meeting (Kyoto, Japan) (EuroMPI/ASIA '14). Association for Computing Machinery, New York, NY, USA, 69-76. https://doi.org/10.1145/2642769.2642785

[23] Intel. 2019. Vtune. https://software.intel.com/en-us/vtune

[24] Andreas Jocksch, Matthias Kraushaar, and David Daverio. [n.d.]. Optimized all-to-all communication on multicore architectures applied to FFTs with pencil decomposition. Concurrency and Computation: Practice and Experience 31, 16 ([n. d.]), e4964. https://doi.org/10.1002/cpe.4964

[25] KAUST Supercomputing Laboratory. 2020. Shaheen II. https://www.hpc.kaust. edu.sa/content/shaheen-ii

[26] Andreas Knüpfer, Christian Rössel, Dieter an Mey, Scott Biersdorff, Kai Diethelm, Dominic Eschweiler, Markus Geimer, Michael Gerndt, Daniel Lorenz, Allen Malony, Wolfgang E. Nagel, Yury Oleynik, Peter Philippen, Pavel Saviankou, Dirk Schmidl, Sameer Shende, Ronny Tschüter, Michael Wagner, Bert Wesarg, and Felix Wolf. 2012. Score-P: A Joint Performance Measurement Run-Time Infrastructure for Periscope,Scalasca, TAU, and Vampir. In Tools for High Performance Computing 2011, Holger Brunst, Matthias S. Müller, Wolfgang E. Nagel, and Michael M. Resch (Eds.). Springer Berlin Heidelberg, Berlin, Heidelberg, 79-91.
[27] Krell Institute. 2020. OpenSpeedshop. https://openspeedshop.org/

[28] Brian Leu, Samar Aseeri, and Benson Muite. 2020. Programs and Sample data for "A Comparison of Parallel Profiling Tools for Programs utilizing the FFT". https: //doi.org/10.5281/zenodo.4032591

[29] N. Li and S. Laizet. 2010. 2DECOMP\&FFT - A highly scalable 2D decomposition library and FFT interface. In Cray User Group 2010 conference.

[30] K. A. Lindlan, J. Cuny, A. D. Malony, S. Shende, B. Mohr, R. Rivenburgh, and C. Rasmussen. 2000. A Tool Framework for Static and Dynamic Analysis of ObjectOriented Software with Templates. In SC '00: Proceedings of the 2000 ACM/IEEE Conference on Supercomputing. 49-49. https://doi.org/10.1109/SC.2000.10052

[31] A. Malony, S. Shende, N. Trebon, J. Ray, R. Armstrong, C. Rasmussen, and M. Sottile. 2005. Performance technology for parallel and distributed component software. Concurrency and Computation: Practice and Experience 17, 2-4 (2005), 117-141. https://doi.org/10.1002/cpe.931

[32] A.V. Mohanan, C. Bonamy, and P. Augier. 2018. FluidFFT: common API (C++ and Python) for Fast Fourier Transform HPC libraries,. https://arxiv.org/abs/1807. 01775

[33] Philip Mucci. 2020. PAPIex. https://bitbucket.org/minimalmetrics/papiexoss/src/master/

[34] B. K. Muite and Samar Aseeri. 2020. Benchmarking Solvers for the One Dimensional Cubic Nonlinear Klein Gordon Equation on a Single Core. In Benchmarking, Measuring, and Optimizing, Wanling Gao, Jianfeng Zhan, Geoffrey Fox, Xiaoyi $\mathrm{Lu}$, and Dan Stanzione (Eds.). Springer International Publishing, Cham, 172-184.

[35] Dmitry Nikitenko, Alexander Antonov, Pavel Shvets, Sergey Sobolev, Konstantin Stefanov, Vadim Voevodin, Vladimir Voevodin, and Sergey Zhumatiy. 2017. JobDigest - Detailed System Monitoring-Based Supercomputer Application Behavior Analysis. In Supercomputing, Vladimir Voevodin and Sergey Sobolev (Eds.). Springer International Publishing, Cham, 516-529.

[36] D. Pekurovsky. 2012. P3DFFT: a framework for parallel computations of Fourier transforms in three dimensions. SIAM fournal on Scientific Computing 34, 4 (08 2012), C192-C209. https://doi.org/10.1137/11082748X

[37] M. Pippig. 2013. PFFT - An Extension of FFTW to Massively Parallel Architectures. SIAM 7. Sci. Comput. 35 (2013), C213 - C236. https://doi.org/10.1137/120885887

[38] S. Plimpton. 2017. Parallel FFT. http://www.sandia.gov/ sjplimp/docs/fft/ README.html

[39] S. Plimpton, A. Kohlmemer, P. Blood, and P. Coffman. 2018. fftmpi. https: //fftmpi.sandia.gov/

[40] A. Pope, D. Daniel, and N. Frontiere. 2017. SWFFT. https://xgitlab.cels.anl.gov/ hacc/SWFFT

[41] M. Rajan. 2007. Experiences with the use of CrayPat in Performance Analysis. In Proceedings Cray User Group. https://cfwebprod.sandia.gov/cfdocs/ CompResearch/docs/rajan_paper_cug07.pdf

[42] Sameer S. Shende and Allen D. Malony. 2006. The Tau Parallel Performance System. The International fournal of High Performance Computing Applications 20, 2 (2006), 287-311. https://doi.org/10.1177/1094342006064482

[43] D. Takahashi. 2003. Efficient implementation of parallel three-dimensional FFT on clusters of PCs. Computer Physics Communications 152, 2 (2003), $144-150$. https://doi.org/10.1016/S0010-4655(02)00818-4

[44] D. Takahashi. 2010. An Implementation of Parallel 3-D FFT with 2-D Decomposition on a Massively Parallel Cluster of Multi-core Processors. Springer Berlin Heidelberg, Berlin, Heidelberg, 606-614. https://doi.org/10.1007/978-3-642-14390-8 63

[45] Dan Terpstra, Heike Jagode, Haihang You, and Jack Dongarra. 2010. Collecting Performance Data with PAPI-C. In Tools for High Performance Computing 2009, Matthias S. Müller, Michael M. Resch, Alexander Schulz, and Wolfgang E. Nagel (Eds.). Springer Berlin Heidelberg, Berlin, Heidelberg, 157-173.

[46] Top 500. 2020. Shaheen. https://www.top500.org/system/176502/

[47] Jeffrey S. Vetter and Michael O. McCracken. 2001. Statistical Scalability Analysis of Communication Operations in Distributed Applications. In Proceedings of the Eighth ACM SIGPLAN Symposium on Principles and Practices of Parallel Programming (Snowbird, Utah, USA) (PPoPP '01). ACM, New York, NY, USA, 123-132. https://doi.org/10.1145/379539.379590

[48] N. J. Wright, K. Füerlinger, and D. Skinner. 2010. Effective Performance Measurement at Petascale Using IPM. In 2010 IEEE 16th International Conference on Parallel and Distributed Systems (ICPADS 2010)(ICPADS), Vol. 00. 373-380. https://doi.org/10.1109/ICPADS.2010.16

\section{A PROFILERS AND TYPICAL RESULTS}

This section contains typical output obtained with the profiling tools.

\section{A.1 IPM}

Example report generated using IPM generated on Vedur when running the Klein Gordon solver using 64 cores with the 2decomp\&FFT library. 


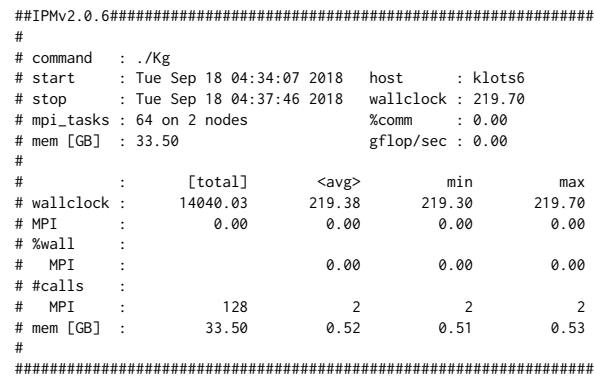

\section{A.2 Scalasca}

Example report generated using Scalasca from a run on Shaheen II when running the Klein Gordon solver using 1024 cores. The first report is for the 2decomp\&FFT library.

$$
\begin{array}{ll}
\text { Estimated aggregate size of event trace: } & 15 \mathrm{MB} \\
\text { Estimated requirements for largest trace buffer (max_buf): } & 15 \mathrm{kB} \\
\text { Estimated memory requirements (SCOREP_TTOAL_MEMORY): } & 4097 \mathrm{kB} \\
\text { (hint: When tracing set SCOREP_TORAL_MEMORYY4097kB to avoid intermediate flushes } \\
\text { or reduce requirements using USR regions filters.) } \\
\text { flt tyype max_buf[B] visits time[s] time[\%] time/visit[us] region }
\end{array}
$$

\begin{tabular}{|c|c|c|c|c|c|c|}
\hline & & & & & \\
\hline ALL & 15,107 & 289,793 & 5055.35 & 100.0 & 17444.68 & ALL \\
\hline MPI & 14,130 & 249,856 & 1989.65 & 39.4 & 7963.18 & MPI \\
\hline USR & 792 & $\quad 32,769$ & 171.31 & 3.4 & 5227.87 & USR \\
\hline $\mathrm{COM}$ & 144 & 6,144 & 2894.37 & 57.3 & 471088.17 & $\mathrm{COM}$ \\
\hline SCOREP & 41 & 1,024 & 0.02 & 0.0 & 20.65 & SCOREP \\
\hline MPI & 11,616 & 180,224 & 1786.14 & 35.3 & 9910.64 & MPI_Alltoall \\
\hline MPI & 792 & 12,288 & 3.04 & 0.1 & 247.41 & MPI_Reduce \\
\hline USR & 720 & 30,720 & 161.56 & 3.2 & 5259.24 & storeold \\
\hline MPI & 462 & 7,168 & 10.77 & 0.2 & 1502.72 & MPI_Allreduce \\
\hline MPI & 384 & 16,384 & 36.63 & 0.7 & 2235.54 & MPI_Cart_sub \\
\hline MPI & 240 & 10,240 & 3.32 & 0.1 & 324.04 & MPI_Cart_create \\
\hline MPI & 192 & 8,192 & 0.00 & 0.0 & 0.46 & MPI_Cart_coords \\
\hline MPI & 144 & 6,144 & 0.01 & 0.0 & 1.97 & MPI_Cart_shift \\
\hline MPI & 132 & 2,048 & 2.36 & 0.0 & 1154.77 & MPI_Bcast \\
\hline COM & 96 & 4,096 & 545.30 & 10.8 & 133129.63 & enercalc \\
\hline MPI & 48 & 2,048 & 0.00 & 0.0 & 0.34 & MPI_Comm_rank \\
\hline MPI & 48 & 2,048 & 0.00 & 0.0 & 0.72 & MPI_Comm_size \\
\hline SCOREP & 41 & 1,024 & 0.02 & 0.0 & 20.65 & KgScalasca \\
\hline COM & 24 & 1,024 & 0.01 & 0.0 & 7.29 & readinputfile \\
\hline USR & 24 & 1 & 0.09 & 0.0 & 91238.32 & saveresults \\
\hline MPI & 24 & 1,024 & 0.00 & 0.0 & 1.21 & MPI_Cart_get \\
\hline USR & 24 & 1,024 & 0.14 & 0.0 & 137.21 & getgrid \\
\hline USR & 24 & 1,024 & 9.52 & 0.2 & 9293.37 & initialdata \\
\hline MPI & 24 & 1,024 & 55.51 & 1.1 & 54204.75 & MPI_Init \\
\hline COM & 24 & 1,024 & 2349.06 & 46.5 & 2294003.24 & $\mathrm{~kg}$ \\
\hline & & & & & & \\
\hline
\end{tabular}

The second report is for the FFTE library.

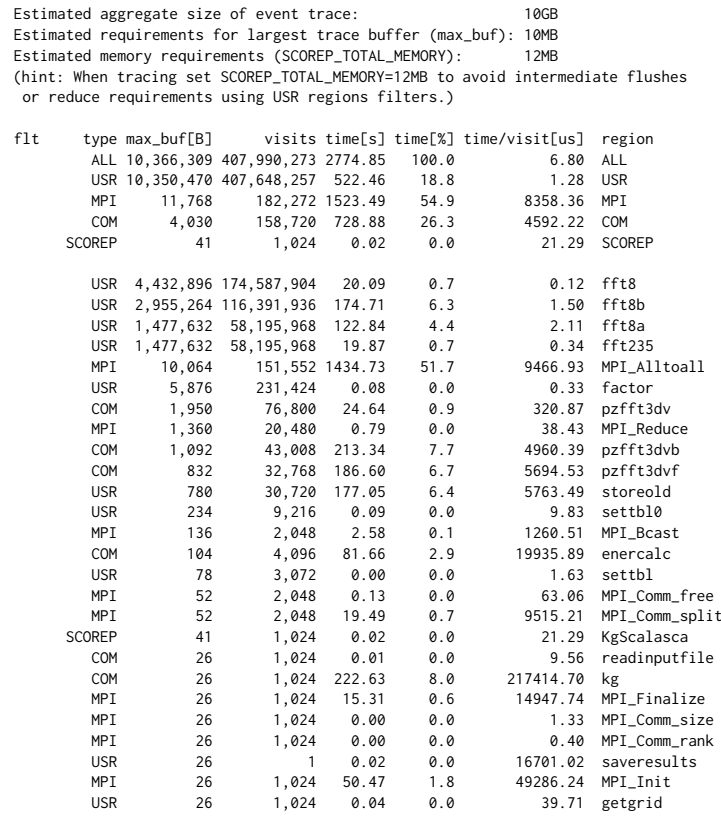

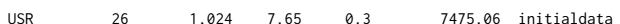

\section{A.3 TAU}

Example report generated using TAU when running the Klein Gor-

\begin{tabular}{|c|c|c|c|c|c|}
\hline \% Time & $\begin{array}{r}\text { Exclusive } \\
\text { msec }\end{array}$ & $\begin{array}{l}\text { Inclusive } \\
\text { total msec }\end{array}$ & \#call & \#Subrs & $\begin{array}{l}\text { Inclusive Name } \\
\text { usec/call }\end{array}$ \\
\hline 100.0 & 26,862 & $1: 25: 09.260$ & 1024 & 1024 & 4989512 . TAU application \\
\hline 99.5 & 155 & $1: 24: 42.397$ & 1024 & 1024 & 4963279 main \\
\hline 99.5 & $38: 54.667$ & $1: 24: 42.241$ & 1024 & 249857 & 4963127 MAIN__- \\
\hline 34.3 & $29: 13.799$ & $29: 13.799$ & 180224 & 0 & 9731 MPI_Alltoall() \\
\hline 14.0 & $8: 11.688$ & $11: 55.319$ & 4096 & 36864 & 174638 enercalc_ \\
\hline 3.7 & $3: 06.982$ & $3: 06.982$ & 1024 & 0 & 182600 MPI_Finalize() \\
\hline 2.9 & $2: 27.637$ & $2: 27.637$ & 30720 & 0 & 4806 storeold_ \\
\hline 1.1 & 55,457 & 55,457 & 1024 & 0 & 54157 MPI_Init() \\
\hline 0.8 & 60 & 40,969 & 1024 & 2048 & 40009 readinputfile \\
\hline 0.8 & 40,908 & 40,908 & 2048 & 0 & 19975 MPI_Bcast() \\
\hline 0.8 & 39,554 & 39,554 & 16384 & 0 & 2414 MPI_Cart_sub() \\
\hline 0.3 & 14,072 & 14,072 & 7168 & 0 & 1963 MPI_Allreduce() \\
\hline 0.2 & 9,968 & 9,968 & 1024 & 0 & 9735 initialdata_ \\
\hline 0.1 & 6,151 & 6,151 & 12288 & 0 & 501 MPI_Reduce() \\
\hline 0.0 & 1,209 & 1,209 & 10240 & 0 & 118 MPI_Cart_create() \\
\hline 0.0 & 46 & 46 & 1024 & 0 & 45 getgrid_ \\
\hline 0.0 & 18 & 18 & 1 & 0 & 18054 saveresults_ \\
\hline 0.0 & 9 & 9 & 8192 & 0 & 1 MPI_Cart_coords () \\
\hline 0.0 & 6 & 6 & 6144 & 0 & 1 MPI_Cart_shift () \\
\hline 0.0 & 1 & 1 & 1024 & 0 & 2 MPI_Cart_get() \\
\hline 0.0 & 0.894 & 0.894 & 2048 & 0 & O MPI_Comm_size() \\
\hline 0.0 & 0.599 & 0.599 & 2048 & 0 & (0 MPI_Comm_rank() \\
\hline 0.0 & 0.384 & 0.384 & 1024 & 0 & 0 MPI_Type_size() \\
\hline
\end{tabular}
don solver using 1024 cores of Shaheen II. Due to space constraints, node level information and a summary of average information

\begin{tabular}{|c|c|c|c|c|c|c|}
\hline \%Time & $\begin{array}{r}\text { Exclusive } \\
\text { msec }\end{array}$ & $\begin{array}{l}\text { Inclusive } \\
\text { total msec }\end{array}$ & \#Call & \#Subrs & $\begin{array}{l}\text { Inclusive } \\
\text { usec/call }\end{array}$ & Name \\
\hline 100.0 & 7 & $49: 20.598$ & 1024 & 1024 & 2891210 & . TAU application \\
\hline 100.0 & $3: 56.906$ & $49: 20.591$ & 1024 & 111617 & 2891203 & KG \\
\hline 72.9 & 24,649 & $35: 58.678$ & 76800 & 306176 & 28108 & PZFFT3DV \\
\hline 46.6 & $22: 59.941$ & $22: 59.941$ & 151552 & 0 & 9105 & MPI_Alltoall() \\
\hline 38.5 & $3: 27.367$ & $18: 59.314$ & 43008 & $3.31162 E+07$ & 26491 & PZFFT3DVB \\
\hline 33.6 & $3: 04.985$ & $16: 34.543$ & 32768 & $2.52314 E+07$ & 30351 & PZFFT3DVF \\
\hline 14.5 & $1: 13.790$ & $7: 09.595$ & 4096 & 32768 & 104882 & ENERCALC \\
\hline 12.2 & 49,956 & $6: 01.563$ & $5.8196 \mathrm{E}+07$ & $1.60597 E+08$ & & FFT235 \\
\hline 7.0 & 25,215 & $3: 26.386$ & $1.02401 \mathrm{E}+08$ & $1.02401 E+08$ & & FFT8 [THROTTLED] \\
\hline 6.8 & $3: 20.838$ & $3: 20.838$ & 1024 & 0 & 196132 & MPI_Finalize() \\
\hline 6.0 & $2: 58.012$ & $2: 58.012$ & 30720 & 0 & 5795 & STOREOLD \\
\hline 5.5 & $2: 41.939$ & $2: 41.939$ & $1.02401 \mathrm{E}+08$ & 0 & & FFT8B [THROTTLED] \\
\hline 4.2 & $2: 04.451$ & $2: 04.451$ & $5.8196 \mathrm{E}+07$ & 0 & & FFT8A \\
\hline 1.8 & 52,073 & 52,073 & 1024 & 0 & 50853 & MPI_Init() \\
\hline 1.2 & 36 & 35,458 & 1024 & 2048 & 34628 & READINPUTFILE \\
\hline 1.2 & 35,422 & 35,422 & 2048 & 0 & 17296 & MPI_Bcast() \\
\hline 0.5 & 16,185 & 16,185 & 2048 & 0 & 7903 & MPI_Comm_split() \\
\hline 0.3 & 7,671 & 7,671 & 1024 & 0 & 7491 & INITIALDATA \\
\hline 0.0 & 859 & 859 & 20480 & 0 & 42 & MPI_Reduce() \\
\hline 0.0 & 8 & 114 & 3072 & 12288 & 37 & SETTBL \\
\hline 0.0 & 104 & 104 & 9216 & 0 & & SETTBLO \\
\hline 0.0 & 74 & 74 & 1024 & 0 & & GETGRID \\
\hline 0.0 & 57 & 57 & 231424 & 0 & & FACTOR \\
\hline 0.0 & 22 & 22 & 1 & 0 & 22157 & SAVERESULTS \\
\hline 0.0 & 18 & 18 & 2048 & 0 & & MPI_Comm_free() \\
\hline 0.0 & 0.642 & 0.642 & 1024 & 0 & & MPI_Comm_size() \\
\hline 0.0 & 0.268 & 0.268 & 1024 & 0 & & MPI_Comm_rank() \\
\hline
\end{tabular}
at a per node level are not presented. The first report is for the 2decomp\&FFT library.

The second report is for the FFTE library.

\section{A.4 Craypat-lite}

Example report generated using Craypat-lite when running the Klein Gordon solver using 1024 cores of Shaheen II. Information on file $\mathrm{I} / \mathrm{O}$ is removed since it is not relevant for this work, similarly much of the information in the first table is replicated in the second table, so the first table is removed. The first report is for the 2decomp\&FFT library.

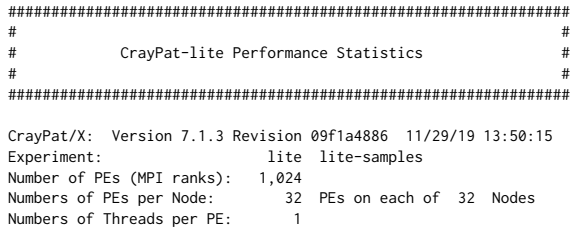




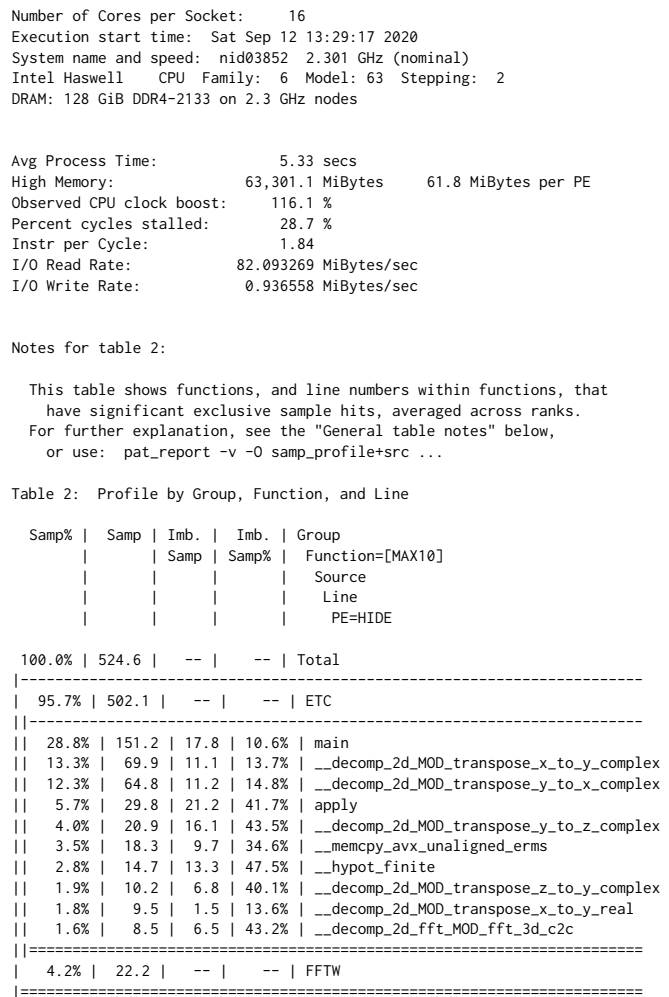

Observation: MPI utilization

No suggestions were made because MPI data was not traced (use pat_build -g mpi)

Observation: Program sensitivity to memory latency

The relatively low memory bandwidth utilization but significant rate of stalls in the program suggest that its performance is limited by

memory latency.
It could be beneficial to improve prefetching in loops in functions high in the profile, by modifying compiler-generated prefetches or inserting directives into the source code.

Notes for table 3:

This table shows memory traffic to local and remote memory for numa nodes, taking for each numa node the maximum value across nodes. and botton 3 node values.

"General table notes" below, or use: pat_report $-\mathrm{v}-0$ mem_bw ...

Table 3: Memory Bandwidth by Numanode

Memory | Local | Remote | Thread | Memory | Memory | Numanode Traffic Memory | Memory | Time | Traffic | Traffic | Node Id=[max3,min3] GBytes | Traffic | Traffic |

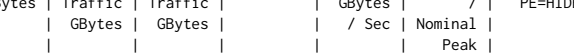

\begin{tabular}{|c|c|c|c|c|c|}
\hline & 28.901 & $28.15 \mathrm{I}$ & $0.75|7.898865|$ & 3.661 & $5.4 \%$ | numanode. 0 \\
\hline & $28.88 ।$ & $28.14 \mid$ & $0.74|5.639828|$ & 5.12 । & 7.5\% | nid. 3864 \\
\hline & $28.88 ।$ & $28.15 \mid$ & $0.72|5.639401|$ & 5.12 I & 7.5\% | nid. 3857 \\
\hline & $28.87 \quad$ & 28.15 । & $0.72|5.642093|$ & 5.12 । & 7.5\% | nid. 3865 \\
\hline & 28.67 । & $27.94 \mid$ & $0.73|5.629921|$ & 5.091 & 7.5\% | nid. 3875 \\
\hline & 28.65 । & $27.92 ।$ & $0.73|5.631063|$ & 5.091 & 7.5\% | nid. 3882 \\
\hline & 28.641 & $27.91 ।$ & $0.72|5.643203|$ & $5.07 \mathrm{I}$ & 7.4\% | nid. 3883 \\
\hline & $28.84 \mid$ & $27.98 ।$ & $0.86|5.737451|$ & 5.031 & 7. $4 \%$ | numanode. 1 \\
\hline & $28.82 ।$ & $27.98 \mid$ & $0.84|5.595931|$ & 5.15 । & 7.5\% | nid. 3856 \\
\hline & $28.82 ।$ & 27.97 । & $0.84|5.595890|$ & 5.151 & 7.5\% | nid. 3872 \\
\hline & $28.80 ।$ & 27.951 & $0.85|5.598326|$ & $5.14 \mathrm{I}$ & 7.5\% | nid. 3873 \\
\hline & $28.59 \mid$ & $27.74 \mid$ & $0.85|5.596116|$ & $5.11 \mathrm{I}$ & 7.5\% | nid. 3875 \\
\hline & $28.56 ।$ & $27.71 \mid$ & $0.85|5.596610|$ & 5.101 & 7.5\% | nid. 3878 \\
\hline & $28.55 \mid$ & $27.72 \mid$ & $0.82|5.602052|$ & $5.10 \mid$ & 7.5\% | nid. 3853 \\
\hline
\end{tabular}

Notes for table 4:
This table shows energy and power usage for the nodes with the maximum, mean, and minimum usage, as well as the sum of usage over maximum,

Energy and power for accelerators is also shown, if applicable.

For further explanation, see the "General table notes" below,

or use: pat_report $-v-0$ program_energy

Table 4: Program energy and power usage (from Cray PM)

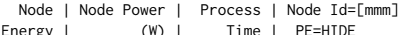

(J)

56,092 | 10,524.474 | 5.329682 | Total

\begin{tabular}{|l|l|l|l|l|l|l|}
1,828 & 343.036 & 5.328890 & nid. 3854
\end{tabular}

\begin{tabular}{|l|l|l|l|l|}
1,756 & 329.623 & 5.327291 & nid. 387
\end{tabular}

\begin{tabular}{ll|l|l|l|l|l|}
1,687 & 316.664 & 5.327410 & nid. 3875
\end{tabular}

Program invocation:

/2decomp/run_craypat_1024/./KgCraypat

$============$ End of CrayPat-lite output

The second report is for the FFTE library.

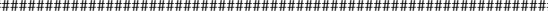

$\begin{array}{lll}\# & \text { CrayPat-lite Performance Statistics } & \# \\ \# & \# \\ \# & \end{array}$

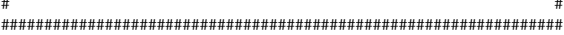

CrayPat/X: Version 7.1.3 Revision ø9f1a4886 11/29/19 13:50:15

Experiment: lite lite-samples

Number of PEs (MPI ranks): 1,024

Numbers of PEs per Node: 32 PEs on each of 32 Nodes

Numbers of Threads per PE: $\quad 1$

Execution start time: Thu Sep 10 17:43:34 2020

Execution start time: Thu Sep 10 17:43:34 2020

System name and speed: nid 07102 2.301 $\mathrm{GHz}$ (nominal)
Intel Haswell CPU Family: 6 Model: 63 Stepping:

DRAM: 128 GiB DDR4-2133 on $2.3 \mathrm{GHz}$ nodes

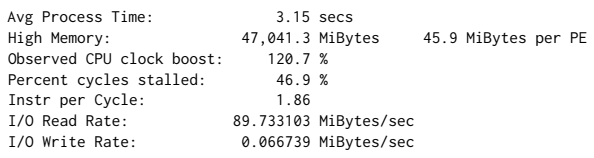

I/O Write Rate: $\quad 0.066739 \mathrm{MiBytes} / \mathrm{sec}$

Notes for table 2:

This table shows functions, and line numbers within functions, that have significant exclusive sample hits, averaged across ranks.

or further explanation, see the "General table notes" below,

or use: pat_report - $v-0$ samp_profile+src ...

Table 2: Profile by Group, Function, and Line

Samp\% | Samp | Imb. | Imb. | Group

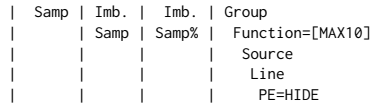

$100.0 \%$ | 306.9 | -- | -- | Total

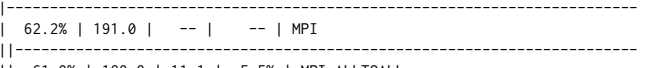

| $61.9 \%$ | $189.9|11.1| 5.5 \%$ | MPI_ALLTOALL

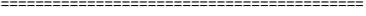

$26.5 \%$ | 81.4 | $\quad--|\quad--|$ USER

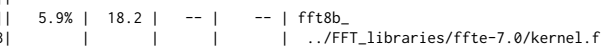

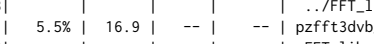

3) | | FFT_libraries/ffte-7.0/mpi/pzfft3dv.f

|l||- $2.3 \%$ | $7.0|6.0| 46.0 \%$ | line. 309

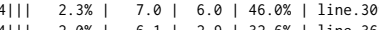

4|| $\mid \quad 2.0 \%$ | 6.1 | 2.9 | $32.6 \%$ | line. 363

11) $5.1 \%+15.7+--\mid$

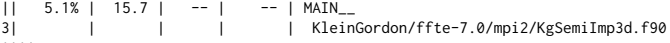

4|| $\mid 2.2 \%$ | 6.6 | 6.4 | $48.9 \%$ | line. 278

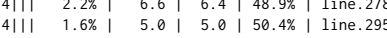

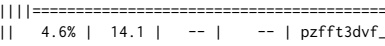

\begin{tabular}{ll|l|l|l|l} 
II & $4.6 \%$ & 14.1 & -- & -- & pzfft3dvf_ \\
$3 \mid$ & & & & &
\end{tabular}

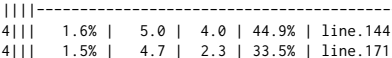

4|| $\mid \quad 1.3 \%$ | 4.0 | $4.0 \mid 50.2 \%$ | line. 198

\begin{tabular}{ll|l|l|l|l}
$\|$ & $4.2 \%$ & 13.0 & -- & -- & fft8a_ \\
$3 \mid$ & & $\mid$ & $\mid$ & |. IFFT_libraries/ffte-7.0/kernel.f
\end{tabular}

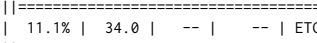

|| $8.9 \%$ | 27.4 | 12.6 | $31.5 \%$ | __memcpy_avx_unaligned_erms

\begin{tabular}{ll|r|r|r|l} 
& \\
11 & $1.5 \%$ & 4.7 & 5.3 & $52.8 \%$ & -_memppy_avx_unaligned_erms \\
\end{tabular} 


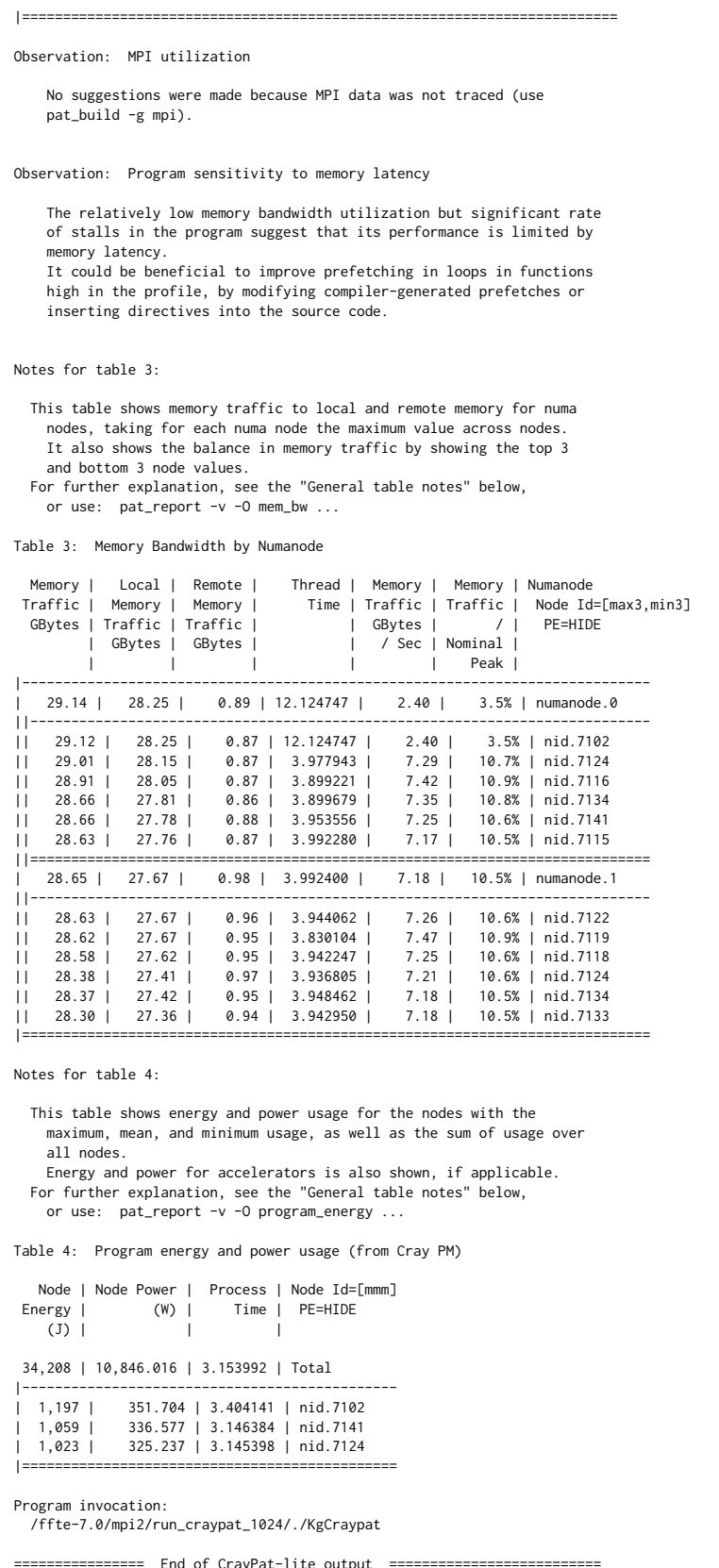

\section{A.5 FPMPI}

Example report generated using FPMPI when running the Klein Gordon solver using 1024 cores of Shaheen II. Information on point to point communications has been removed since for this application all communication calls are collective. The first report is for the 2decomp\&FFT library.

MPI Routine Statistics (FPMPI2 Version 2.4) Options: FPMPI enabled, Collective sync, Explanation of data:

Times are the time to perform the operation, e.g., the time for MPI_Send Average times are the average over all processes, e.g., sum (time on each process) number of processes

Min and max values are over all processes

(Data is always average/min/max)

Amount of data is computed in bytes. For point-to-point operations,

it is the data sent or received. For collective operations, it is the

\begin{abstract}
data contibuted to the operation. E.g., for an MPI_Bcast, the amount of data is the number of bytes provided by the root, counted only at the root. For synchronizing collective operations, the average, $\min$, and max time spent synchronizing is shown next.

Calls by message size shows the fraction of calls that sent messages of a particular size. The bins are
0 bytes, $1-4$ bytes, $5-8$ bytes, $9-16,17-32,33-64,-128,-256,-512,-1024$ $-4 \mathrm{~K},-8 \mathrm{~K},-16 \mathrm{~K},-32 \mathrm{~K},-64 \mathrm{~K},-128 \mathrm{~K},-256 \mathrm{~K},-512 \mathrm{~K},-1 \mathrm{M},-4 \mathrm{M},-8 \mathrm{M},-16 \mathrm{M}$, $-4 \mathrm{~K},-8 \mathrm{~K},-16 \mathrm{~K},-32 \mathrm{~K},-64 \mathrm{~K},-128 \mathrm{~K},-256 \mathrm{~K},-5-6$
$-32 \mathrm{M},-64 \mathrm{M},-128 \mathrm{M},-256 \mathrm{M},-512 \mathrm{M},-1 \mathrm{~GB},>1 \mathrm{~GB}$.

Each bin is represented by a single digit, representing the 10 's of percent of messages within this bin. A $\theta$ represents precisely $\theta$, a . (period) represents more than $\theta$ but less than $10 \%$. A * represents $100 \%$. Messages by message size shows similar information, but for the total message size.
\end{abstract}

The experimental topology information shows the 1 -norm distance that the longest point-to-point message travelled, by process.

MPI_Pcontrol may be used to control the collection of data. Use the values defined in fpmpi.h, such as FPMPI_PROF_COLLSYNC, to control what data is collected or reported by FPMPI2.

Date:

Processes: $\quad 1024$

Timing Stats: [seconds] [min/max] [min rank/max rank]

[min rank/max rak
wall-clock: $5.427 \sec 5.420879 / 5.899858 \quad 659 / \theta$

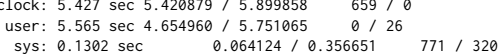

Memory Usage Stats (RSS) [min/max KB]: 78992/81152

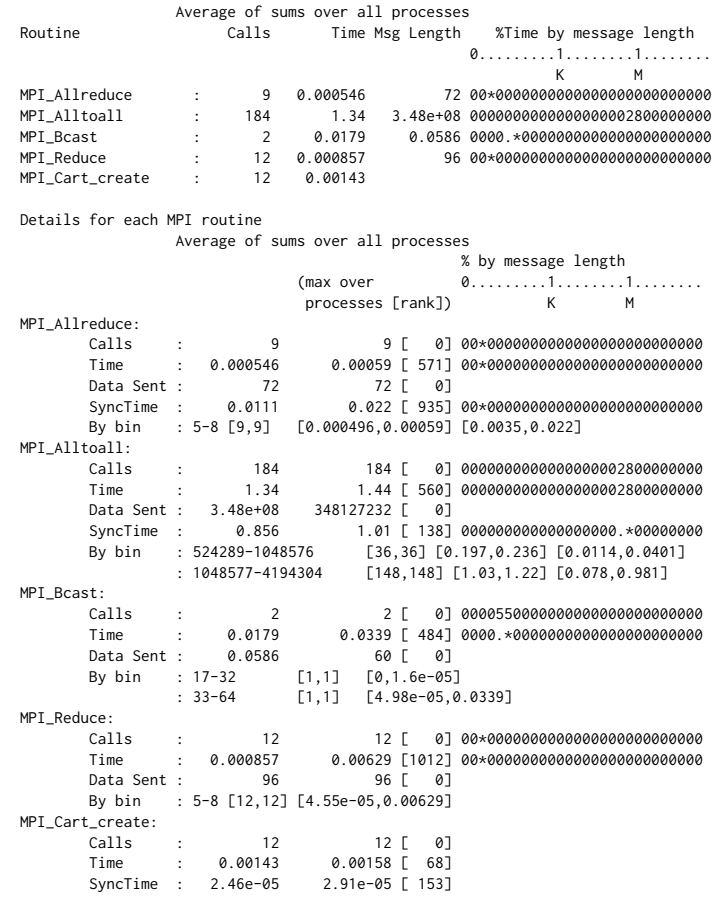

PAPI Data:
PAPI_TOT_CYC: average $=15447837144.74, \max =15528701710, \min =12149808092$ PAPI_L3_TCM: average $=8980659.27, \max =9567080, \min =8494555$

PAPI_L2_DCA: average $=150172977.33, \max =206534513, \min =145189127$

The second report is for the FFTE library.

MPI Routine Statistics (FPMPI2 Version 2.4)

Options: FPMPI enabled, Collective sync,

Times are the time to perform the operation, e.g., the time for MPI_Send Average times are the average over all processes, e.g., sum (time on each process) / number of processes

Min and max values are over all processes

(Data is always average/min/max)

Amount of data is computed in bytes. For point-to-point operations, it is the data sent or received. For collective operations, it is the data is the number of bytes provided by the root, counted only at the root. For synchronizing collective operations, the average, min, and max time spent synchronizing is shown next.

Calls by message size shows the fraction of calls that sent messages of a particular size. The bins are

0 bytes, $1-4$ bytes, $5-8$ bytes, $9-16,17-32,33-64,-128,-256,-512,-1024$ $-4 \mathrm{~K},-8 \mathrm{~K},-16 \mathrm{~K},-32 \mathrm{~K},-64 \mathrm{~K},-128 \mathrm{~K},-256 \mathrm{~K},-512 \mathrm{~K},-1 \mathrm{M},-4 \mathrm{M},-8 \mathrm{M},-16 \mathrm{M}$, $-32 \mathrm{M},-64 \mathrm{M},-128 \mathrm{M},-256 \mathrm{M},-512 \mathrm{M},-1 \mathrm{~GB},>1 \mathrm{~GB}$.

Each bin is represented by a single digit, representing the $10^{\prime}$ 's of percent 


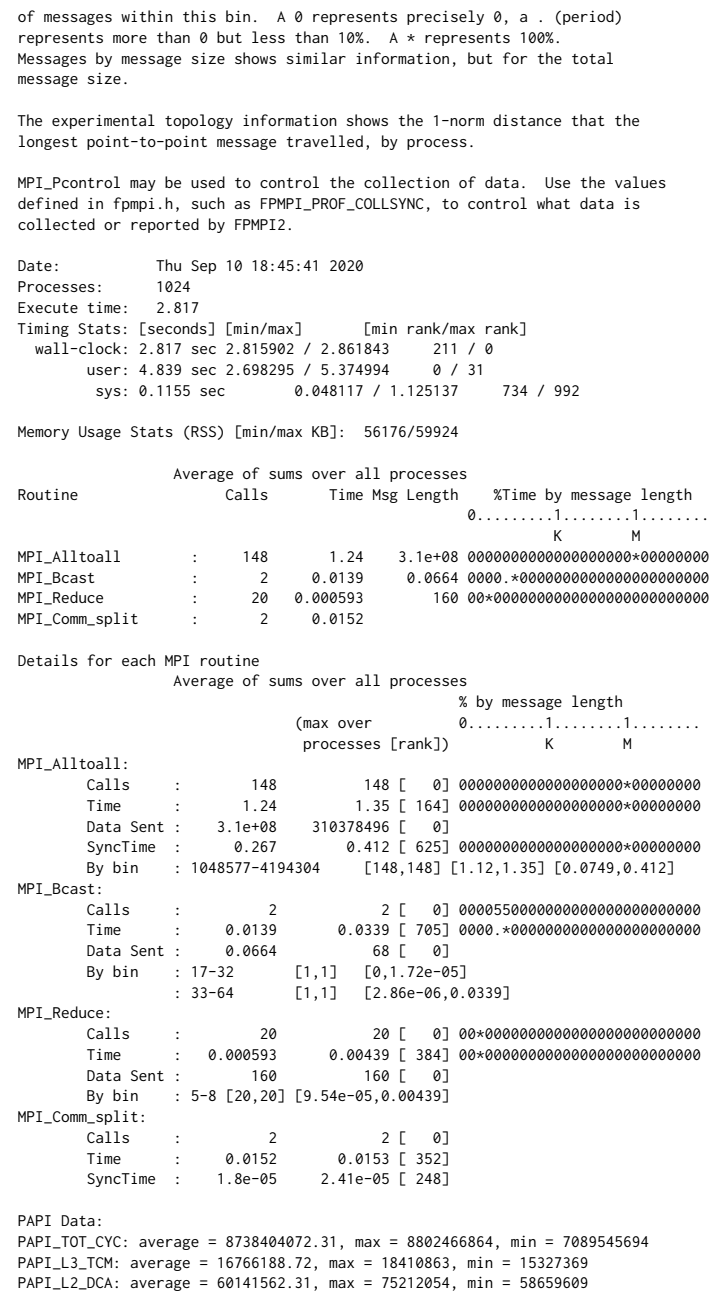

\section{A.6 mpiP}

Example report generated using mpiP when running the Klein Gordon solver using 1024 cores of Shaheen II. Due to space constraints, only aggregate time information is provided. Information at a node level is removed. The first report is for the 2decomp\&FFT library.

a mpip

$\begin{array}{ll}\text { @ mpiP } & \\ \text { @ Command : /2decomp/summary_run_mpip_1024/./KgmpiP } \\ \text { @ Version } & : 3.4 .2 \\ \text { @ MPIP Build date } & : \text { Aug } 16 \quad 2020,11: 18: 40 \\ \text { @ Start time } & : 202009 \quad 1505: 29: 36 \\ \text { @ Stop time } & : 202009 \quad 1505: 29: 41 \\ \text { @ Timer Used } & : \text { PMPI_Wtime } \\ \text { @ MPIP env var } & : \text { [null] } \\ \text { @ Collector Rank } & : 0 \\ \text { @ Collector PID } & : 10192 \\ \text { @ Final Output Dir } & : \\ \text { @ Report generation } & : \text { Single collector task }\end{array}$

-

Call $\quad$ Site Time App\% MPI\% Count

$\begin{array}{lrrrrrr}\text { Call } & \text { Site } & \text { Time } & \text { App\% } & \text { MPI\% } & \text { Count } & \text { CoV } \\ \text { Alltoall } & 11 & 6.76 \mathrm{e}+05 & 14.20 & 34.99 & 32768 & 0.08\end{array}$

$\begin{array}{lllllll}\text { Alltoall } & 11 & 6.76 \mathrm{e}+05 & 14.20 & 34.99 & 32768 & 0.08 \\ \text { Alltoall } & 10 & 6.31 \mathrm{e}+05 & 13.27 & 32.70 & 43008 & 0.05\end{array}$

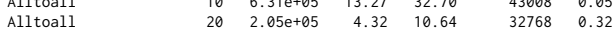

$\begin{array}{lllllll}\text { Alltoall } & 28 & 9.88 \mathrm{e}+04 & 2.08 & 5.12 & 43008 & 0.10\end{array}$

$\begin{array}{lrrrrrr}\text { Bcast } & 7 & 7.81 \mathrm{e}+04 & 1.64 & 4.04 & 1024 & 0.03\end{array}$

$\begin{array}{llllllll}\text { Alltoall } & 6 & 6.55 \mathrm{e}+04 & 1.38 & 3.39 & 7168 & 0.09\end{array}$

$\begin{array}{llllllll}\text { Alltoall } & 29 & 5.67 \mathrm{e}+04 & 1.19 & 2.94 & 7168 & 0.06\end{array}$

$\begin{array}{lrrrrrr}\text { Alltoall } & 15 & 3.42 \mathrm{e}+04 & 0.72 & 1.77 & 7168 & 0.19 \\ \text { Cart_sub } & 8 & 2.95 \mathrm{e}+04 & 0.62 & 1.53 & 7168 & 0.00\end{array}$

$\begin{array}{lrrrrrr} & 8 & 2.95 \mathrm{e}+04 & 0.62 & 1.53 & 7168 & 0.00 \\ \text { Alltoall } & 5 & 2.6 \mathrm{e}+04 & 0.55 & 1.35 & 7168 & 0.02\end{array}$

$\begin{array}{lrrrrrr}\text { Allreduce } & 17 & 1.21 \mathrm{e}+04 & 0.25 & 0.63 & 7168 & 0.33 \\ \text { Cart_sub } & 22 & 7.86 \mathrm{e}+03 & 0.17 & 0.41 & 7168 & 0.01\end{array}$

$\begin{array}{lrrrrrr}\text { Reduce } & 19 & 4.05 \mathrm{e}+03 & 0.09 & 0.21 & 4096 & 1.09 \\ \text { Cart_sub } & 2 & 3.74 \mathrm{e}+03 & 0.08 & 0.19 & 1024 & 0.00 \\ \text { Cart_create } & 26 & 992 & 0.02 & 0.05 & 7168 & 0.05 \\ \text { Cart_sub } & 13 & 186 & 0.00 & 0.01 & 1024 & 0.01 \\ \text { Cart_create } & 21 & 127 & 0.00 & 0.01 & 1024 & 0.04 \\ \text { Cart_create } & 12 & 116 & 0.00 & 0.01 & 1024 & 0.05 \\ \text { Reduce } & 27 & 81.6 & 0.00 & 0.00 & 4096 & 1.31 \\ \text { Cart_create } & 4 & 55.7 & 0.00 & 0.00 & 1024 & 0.10\end{array}$

The second report is for the FFTE library.

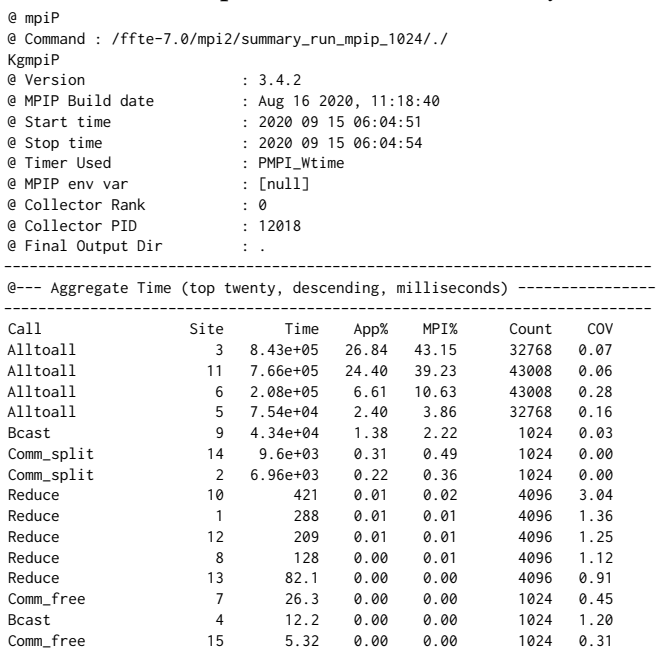

